\title{
MULTIPLE STARS WITH BOTH INNER AND OUTER ORBITS OBSERVED SPECTROSCOPICALLY
}

\author{
R. F. Griffin ${ }^{1}$ \\ RESUMEN \\ Reportamos avances en la determinación de las órbitas exteriores, de largo período, en algunas estrellas triples \\ espectroscópicas.
}

\section{ABSTRACT}

Some progress has been made towards determining the long-period outer orbits of certain spectroscopic multiple stars.

Key Words: BINARIES: SPECTROSCOPIC

\section{INTRODUCTION}

Many examples are known of 'visual' multiple stars, with three or more components in hierarchical orbits. Many spectroscopic binaries, too, are components of visual double or multiple stars. Relatively few 'spectroscopic multiples' are known, however, in which both the inner (short-period) and outer (longperiod) orbits have been documented spectroscopically.

The principal reason for that state of affairs is easy to see: radial-velocity studies have not been systematically pursued for long enough for the outer orbits of the majority of multiple systems to have been seen round a complete cycle. Moreover, the velocity amplitudes at long periods are necessarily small, and observers are disinclined to spend a lifetime measuring tiny drifts in the radial velocities of long-period objects when the sky is full of un-studied (and indeed of as-yet-undiscovered) bright short-period systems of large amplitude that can be documented much more easily and quickly. Furthermore, quite apart from their personal interests and inclinations, many observers are at the mercy of telescope-time allocation committees that simply will not grant time, year after year, for projects that in many cases may not come to fruition even within the observer's lifetime let alone before the next meeting of the committee!

The author has made a practice of keeping under observation all objects whose radial velocities he has found to be variable. While not neglecting shortperiod binaries, therefore, he has equally maintained a watch on systems that prove to be of long period. Even so, in many cases the observations do not cover the whole outer orbits of multiple stars, and sometimes all that has been seen is a steady drift in the

\footnotetext{
${ }^{1}$ University of Cambridge, UK. email: rfg@ast.cam.ac.uk.
}

$\gamma$-velocity. This paper, therefore, is necessarily in the nature of a progress report. Even so, some of the objects have been under observation for about 30 years; it is not lack of patience but of longevity that will soon bring this project to a close so far as the author is concerned. He must plead, too, that before he could even start the measurements he was obliged first to develop the observational technique that allows many objects to be kept under routine surveillance with only modest telescopic power and institutional commitment!

\section{SOME EXAMPLES}

It should be pointed out that it does not follow that, just because a system is composed of three or more stars, all of them will be visible in the spectrum: in many cases only one velocity is measureable but it changes with two periods. That is the case for two systems that have recently been formally written up and the papers submitted [added post-conference: and their references can now be given], as follows: HD 100054 B (Ginestet et al. 2003) and HD 191588 (Griffin, Ginestet and Carquillat 2003). The majority of the observations in those papers have been made not by me but by my collaborators, who also discovered the variability of the velocities in the first place.

The short-period orbits of four other systems have already featured in the author's series in The Observatory, where the drifts in the $\gamma$-velocities were noted: HD 7426 (Griffin 1988, paper 80), HR 965 (Griffin 1989, paper 88), HR 2879 B (Griffin 1994, paper 119) and 24 Aquarii (Griffin et al. 1996, paper 128). $24 \mathrm{Aqr}$ is visibly double to expert observers, and its outer orbit has been determined visually; in the other three cases the stars are visually single, but 
enough progress has been made with the radial velocities to hazard preliminary outer orbits, although in the case of HD 7426 that has been possible only under the constraint of arbitrarily fixing the period. HR 2879 B (three members, all visible in the spectrum, of a system that is at least quintuple) underwent quite an exciting periastron passage in its outer orbit a few years ago.

Other objects that are under observation at Cambridge and show periodic velocity changes and also drifts in their $\gamma$-velocities include HR 4454 and HD 112914 (each of which gives a single velocity) and HD 117078 (two velocities that show different short periods and drift in oppositedirections).

\section{REFERENCES}

Ginestet, N., Prieur, J.-L., Carquillat J.-M. \& Griffin, R. F. 2003, MNRAS, 342, 61

Griffin, R. F. 1988, The Observatory, 108, 90

Griffin, R. F. 1989, The Observatory, 109, 180

Griffin, R. F. 1994, The Observatory, 114, 268

Griffin, R. F., Carquillat, J.-M., Ginestet, N. \& Udry, S. 1996, The Observatory, 116, 162

Griffin, R. F., Ginestet, N. \& Carquillat J.-M. 2003, Romanian Astronomical Journal, 13, 31 